\title{
ASSESSMENT OF THE INCIDENCE AND PREVALENCE OF DRY EYES IN DIABETIC INDIVIDUALS AND IT'S EFFECT ON VISUAL ACUITY
}

\author{
MIDHUNA K. ${ }^{1}$, DIVYA N. ${ }^{2 *}$, V. PANIMALAR A. VEERAMANI ${ }^{2}$, BINDU BHASKARAN ${ }^{3}$ \\ 1,2,3 Department of Ophthalmology, Saveetha Medical College and Hospital, Chennai \\ Email: divya.q7@gmail.com
}

Received: 15 Jul 2020, Revised and Accepted: 08 Sep 2020

\begin{abstract}
Objective: To find out the incidence and prevalence of Dry Eyes in Diabetic patients and find out its effect on visual acuity.
\end{abstract}

Methods: A prospective study was conducted in Saveetha Medical College and Hospital. Schirmer's test was done for 100 diabetic patients taking part in the research. Those with dry eyes were tested for their visual acuity and were asked to fill a questionnaire based on associated ocular symptoms. The collected data were analysed using Microsoft Excel.

Results: There is about an incidence of $82 \%$ of dry eyes in diabetic patients. It's more prevalent among elderly women. There seems to be a significant effect of dry eys on visual acuity as $31 \%$ of those tested with dry eyes have a moderate visual impairment.

Conclusion: People with diabetes are at a higher risk of developing dry eye. So diabetic patients should undergo regular ocular examination and must be screened for dry eyes. Early diagnosis and treatment of dry eyes is the best choice to prevent ocular complications in the future.

Keywords: Dry Eye, Tear deficiency, Lacrimnal function unit, Schirmer's Test, Visual acuity

(C) 2020 The Authors. Published by Innovare Academic Sciences Pvt Ltd. This is an open access article under the CC BY license (http://creativecommons.org/licenses/by/4.0/) DOI: http://dx.doi.org/10.22159/ijcpr.2020v12i6.40301. Journal homepage: https://innovareacademics.in/journals/index.php/ijcpr

\section{INTRODUCTION}

According to the Dry Eye Workshop (1995) Dry Eye is a multifactorial disorder of the tear film either due to tear deficiency or excessive tear evaporation characterized by ocular discomfort, visual disturbance and potential damage to the ocular surface [1]. Diabetes is identified as one of the the most common systemic risk factors for Dry Eyes. From the Global Fact Sheet (2019) India has about 77 million adults with diabetes and it is the second country after China with the largest Diabetic population [2]. Based on a study by Hom and De Land $53 \%$ of the individuals with diabetes or borderline diabetes were reported to have Dry Eyes [3].

Tear film hyperosmolarity and Tear film instability are the two major mechanisms by which Diabetes leads to Dry eyes [4]. In some cases, the patients may not be aware of the dry eye symptoms because of decreased corneal sensation [5]. Dry Eye disease also affects the patient's quality of life by affecting the ability to perform day to day activities such as reading, driving and computer-related work that require visual attention [6]. So the aim of the study is to assess the incidence of dry eye among people with diabetes mellitus and find out its effect on visual acuity. This study will prove the necessity for early diagnosis and treatment of dry eyes.

\section{MATERIALS AND METHODS}

The approval for conducting this study was obtained from the Institutional Ethics Committee prior to the study

It is a Prospective type of hospital-based Study. The study was conducted for a duration of 3 mo from January 2020 to March 2020 at Saveetha Medical College and Hospital, a tertiary care teaching hospital in Thandalam, Chennai. All Diabetic patients of either sex from both In-Patient and Out-Patient Departments were included. All Non Diabetic Patients, even those with symptoms of dry eye were excluded. A Sample size of 100 was taken. Patient's demographic data was recorded. Schirmer's Test was performed on all the diabetic patients participating in the research. It is a test that measures the quantity of tears produced in a fixed time. Whatman
41 filterpaper was used to test. After 5 min wetting of filter paper from the bent end is measured. The values were noted for both the eyes. Based on schirmer's test those patients presenting with dry eyes including those with mild dry eyes were tested for their visual acuity using snellen chart. A questionnaire was prepared based on associated ocular symptoms and distributed among these patients.

\section{Statistical analysis}

The data collected was analysed using Microsoft Excel. The results were pictorically expressed in the form of Pie Chart, Percentage, Bar Graph and tabular columns.

\section{RESULTS}

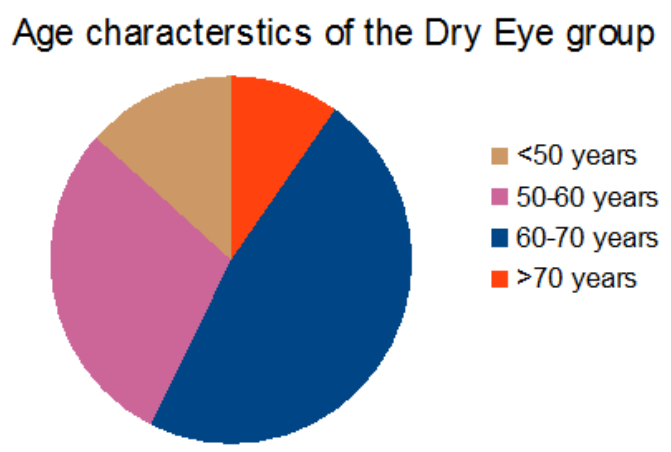

Fig. 1: Age characterstics

The (fig. 1) represents the age distribution of the people who were with dry eye. 11 patients were less than $50 \mathrm{y}, 24$ patients were between 50-60years, 39 patients were between $60-70 \mathrm{y}, 8$ patients are above $70 \mathrm{y}$. It is seen that among the Diabetic patients tested with dry eyes most of them belonged to the age group 60-70 y. 
Table 1: Gender characterstics

\begin{tabular}{llll}
\hline Gender & Total & Dry eye & Non dry eye \\
\hline MALE & 39 & 26 & 13 \\
FEMALE & 61 & 56 & 5 \\
\hline
\end{tabular}

The (table 1) is about the gender characterstics of all the participants of the research and they are characterised into dry eye and non-dry eye group. More number of women (91\%) than men (66\%) are tested with dry eyes proving that women are at a higher risk.

Table 2: Schirmer's test

\begin{tabular}{|c|c|c|}
\hline Type & Range & $\%$ of patients \\
\hline Normal & $>10 \mathrm{~mm}$ & 18 \\
\hline Mild & $8-10 \mathrm{~mm}$ & 24 \\
\hline Moderate & $5-7 \mathrm{~mm}$ & 43 \\
\hline Severe & $<5 \mathrm{~mm}$ & 15 \\
\hline
\end{tabular}

The (table 2) represents the results of the schirmer's test. Based on the values obtained on the schirmer's strip it was categorised into normal $(>10 \mathrm{~mm})$, mild $(8-10 \mathrm{~mm})$, moderate $(5-7 \mathrm{~mm})$, severe $(<5 \mathrm{~mm})$. The number of people belonging to each category was represented as percentages. It is observed that $82 \%$ of the patients have mild to moderate dry eyes proving diabetes as a common risk factor.

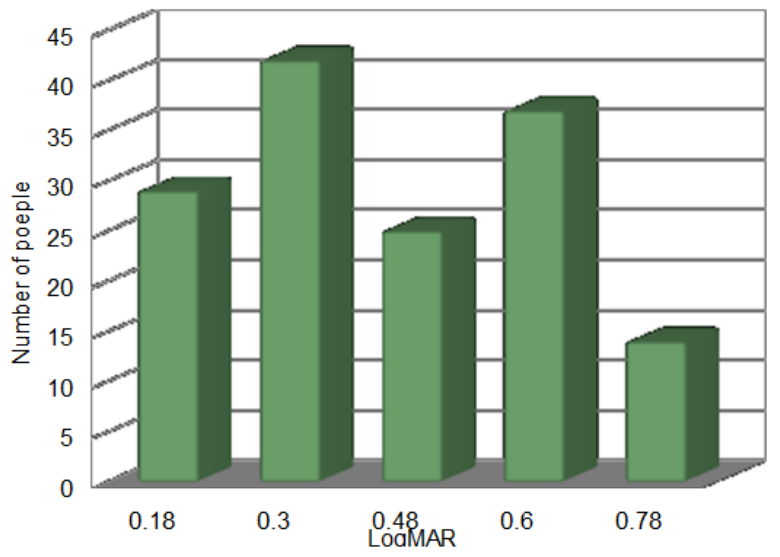

Fig. 2: Visual acuity

Out of the 100 participants, 82 of them have dry eyes including those with mild dry eyes

Visual acuity was measured for those 82 patients (164 eyes).

Visual acuity was measured using snellen chart and the data was collected using Feet as units and was converted into standard LogMAR (Mean Angle of Resolution) for convinience. In fig. 2 the $x-$ axis represents LogMAR and the y-axis refers to the number of people in each scale. It is seen that $31 \%$ of the patients with dry eyes have moderate visual impairment proving that there is an effect of dry eyes on visual acuity.

Patients with dry eyes in addition to visual impairment, come with other complaints of some associated ocular symptoms. This includes dryness, redness, grittiness, irritation and lacrimation, which was represented along the $\mathrm{x}$-axis in the fig. 3 and the number of people suffering from each symptom was represented along the y-axis. Dryness and Grittiness seems to be the most common ocular symptoms among the patients in this study.

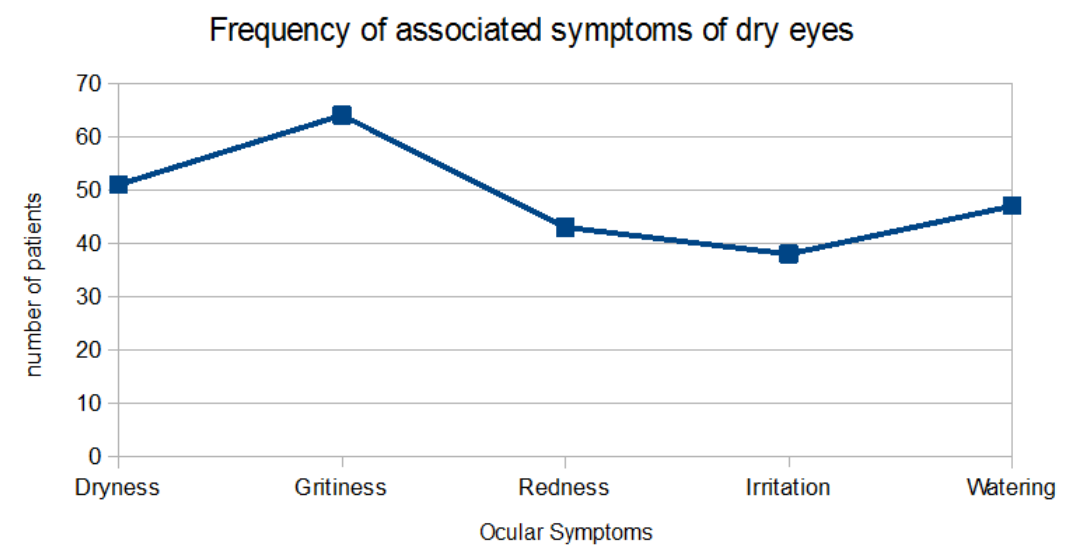

Fig. 3: Associated ocular symptoms 


\section{DISCUSSION}

Diabetes is one of the most commonly found systemic disorders present in almost all households. It is found that people with diabetes have an increased risk of developing dry eyes. Since most of the dry eye symptoms are associated with only mild ocular discomfort, people avoid reporting to the doctors, unaware of the consequences. So this prospective study was conducted to create awareness amongst patients, health care profesionals and researchers about the association between diabetes and dry eyes.

Dry eyes seems to be more prevalent among patients of age group 60-70 y and incidence is more in women than in men. The demographic characterstics of the dry eye patients was similar to the studies done by Yang et al. [7] and Silviana S kamel et al. [8]. The reason behind this is aging results in acinar degeneration and nuclear abnormalities, which causes changes in in Lacrimal Function Unit as a whole. An intact and efficient lipid layer in the tear film is required to prevent the evaporative loss of tears. The lipid layer is thinner and less efficient in older individuls. Aging also results in decreased corneal sensation affecting the reflex iduced lacrimal secretion. One of the other major cause is polypharmacy. Some of the medications have secondary ocular drying effects thus predisposing to dry eye [9]. Androgens regulates the secretory function of Lacrimal Gland. Serum level of Dihydroepiandrosterone, which is one of the main adrenal androgen, is low in women than in men. In addition postmenopausal women have low levels of estrogen, which is known to stimulate the meibomian gland [10]. These factors results in inadequate tear secretion and explains the reason for more prevalence in women than in men.

In this study the incidence of dry eyes in diabetic patients is $82 \%$, which is drastically different from studies by Hom and De Land [3] and Manaviat et al. [11] conducted where the incidence was only around $54 \%$. This may be due to Berkesonian bias, where the results of the research is influenced by different rates of admission in the hospital [12]. Despite the differences in the incidence rate it is proven that diabetes is said to have an influence of dry eyes. Diabetes causes corneal lesions such as superficial punctate keratopathy, trophic ulcers, persistent epithelial defects and recurrent corneal erosions. This leads to lacrimal unit dysfunction resulting in Tear hyperosmolarity and tear film instability. This further interferes with the blink rate, tear secretion, tear film composition thus affecting the normal physiology of the ocular surface that leads to symptoms of ocular discomfort and visual distrurbances [4].

Dry Eye is associated with some ocular symptoms such as dryness, redness, watering, itching and gritiness. Based on the questionnaire answered by the participant's dryness and grittiness is the most common symptom in our study. The dry eye symptoms varies from person to person. They may not experience relief with the same the set of treatments. So the patient must be educted on early dry eye symptoms, relief measures and when to consult a doctor to prevent the ocuurence of complications. From our study it is found out that $31 \%$ of the patients with dry eyes have moderate visual impairment. This is because tear film is said to have a refractive function. The tear film breaks up at a rapid rate in dry eye patients, thus affecting the visual acuity [13].

\section{CONCLUSION}

From this study it is confirmed that diabetes is one of the common systemic risk factors of dry eyes. The prevalence is more among elderly women due to age-related changes and hormonal causes. It is also found that there exist an association between dry eyes and visual impairment. Dry eyes increases the risk of infection due to inadequate tears. If left untreated it may result in infectious keratitis, corneal abrasions, corneal ulceration, which may subsequently lead to loss of vision [14]. It is suggested that all diabetes patients are screened for dry eyes but diabetes is a very common metabolic disorder and such a wide range of screening is not possible. So all those diabetic patients with poor metabolic control, diabetic neuropathy and advanced stages of diabetic retinopathy should be screened for changes in the tear film and ocular surface [15]. The mainstay of treatment for mild and moderate tear deficiency includes tear substitues. In cases of failure of meadical treatment, punctal occlusion can be done to conserve tears. In severe cases, tarsorrhaphy is performed to reduce tear evaporation by reducing the palpebral fissure [16]. So our main aim is to alleviate dry eye symptoms, improve visual function and prevent damage to the ocular surface. Thus early diagnosis and treatment of dry eyes is the best way to prevent further visual impairment and other ocular complications.

\section{ACKNOWLEDGEMENT}

Nil

\section{FUNDING}

Nil

\section{AUTHORS CONTRIBUTIONS}

All the authors have contributed equally.

\section{CONFLICT OF INTERESTS}

\section{Declared none}

\section{REFERENCES}

1. The Ocular Surface. A journal of review linking laboratory science, clinical science, and clinical practice; 2007.

2. International diabetic federation (IDF DIABETIC ATLAS) $9^{\text {th }}$ edition; 2019.

3. Hom, De Land. Self-reported dry eyes and diabetic history. Optometry 2006;77:554-8.

4. Xinyuan Zhang, Lin Zhao, Shijing Deng, Xuguang Sun, Ningli Wang. Dry eye syndrome in patients with diabetic mellitus: prevalance, etiology and clinical characterstics. J Ophthalmol 2016. DOI:10.1155/2016/8201053

5. Jeanne Gonzalez. Patients with diabetes at a risk of dry eye syndrome; 2005.

6. L Tong, S Waduthantri, TY Wong. Impact of dry eye on vision related activities: the Singapore malay eye study. Eye 2010;24:1486-90.

7. Risk factors of dry eye syndrome in chinese: a retrospective case-control study. Optometry Vision Sci 2015;92:199-205.

8. Silviana S Kamel, Thanaa H Mohammed, Yasser A El Zankalony, Ali H Saad. Prevalance of dry eyes in diabetics. J Egyptian Ophthalmol 2017;110:77-82.

9. RS Uma Devi, MS Smitha. Dry eye in diabetic mellitus patienyts and its relationship with diabetic retinopathy. Int J Sci Study 2016;4:67-72.

10. Mark B Abelson, Lisa Lines. Hormones in dry eye: a delicate balance. Rev Ophthalmol 2020;XXVII:1-76.

11. Manaviat MR, Rashidi M, Afkhami Ardekani M, Shoja MR. Prevalance of dry eye and diabetic retinopathy in type 2 diabetic patients. BMC Ophthalmol 2008;8:10.

12. Park Textbook of Social and Preventive Medicine. $25^{\text {th }}$ edition. chapter 3; 2019. p. 81.

13. Kaido M, Uchino M, Yokoi N, Uchino Y, Dogru M, Kawashima M, et al. Dry eye screening by using a functional visula acuity measurement system: the Osaka Study. Investigative Ophthalmol Visual Sci May 2014;55:3275-81.

14. Mark I Golden, Jay J Meyer, Bhupendra C Patel. Dry eye syndrome 2020.

15. Kyung Yun Kook, Rujun Jin, Lan Li, Hyeon Jeong Yoon, Kyung Chul Yoon. Tear osmolarity and matrix metallopeptidase-9 in dry eye associated with Sjogren's syndrome. Korean J Ophthalmol 2020;34:179-86.

16. Ankita S Bhavsar, Samir G Bhavsar, Sunita M Jain. A review on recent advances in dry eye. Pathog Manag 2011;4:50-6. 